\title{
Migratory Insertion of Acetylene in N-Heterocyclic Carbene Complexes of Ruthenium: Formation of (Ruthenocenylmethyl)imidazolium Salts
}

Eva Becker, Verena Stingl, Georg Dazinger, Kurt Mereiter, and Karl Kirchner*

Institute of Applied Synthetic Chemistry and Institute of Chemical Technologies and Analytics, Vienna University of Technology, Getreidemarkt 9, A-1060 Vienna, AUSTRIA

\section{Supporting Information - Crystallographic details}

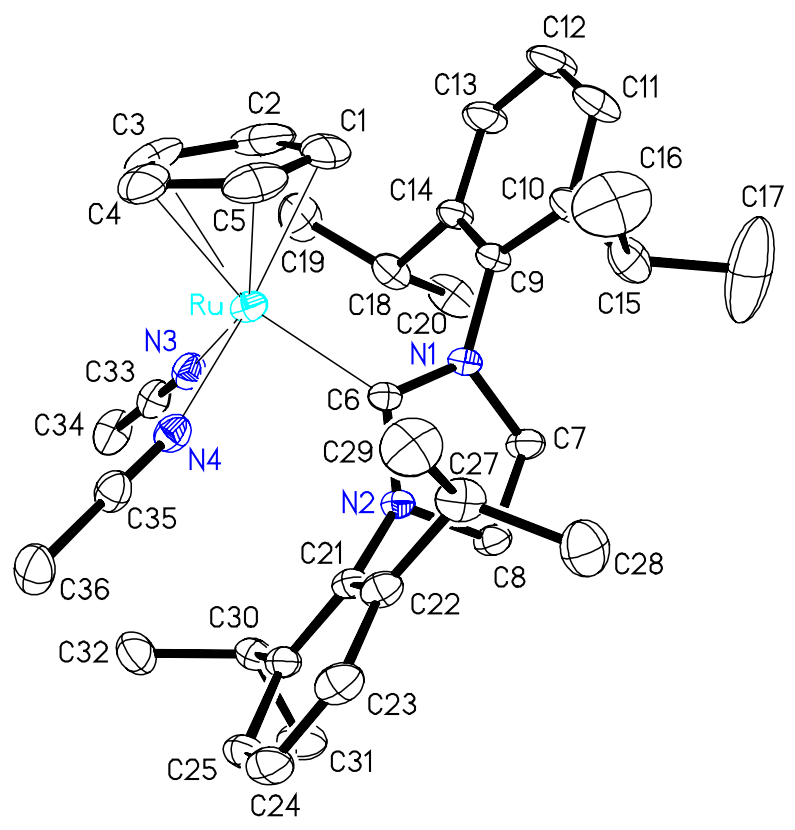

Figure S1. Structural view of $\left[\mathrm{RuCp}\left(\mathrm{CH}_{3} \mathrm{CN}\right)_{2}(\mathrm{SIPr})\right] \mathrm{PF}_{6}$ (1b) ( $\mathrm{SIPr}=1,3-\mathrm{bis}(2,6$-diisopropylphenyl)-4,5dihydroimidazol-2-ylidene) showing $30 \%$ thermal ellipsoids ( $\mathrm{H}$ atoms and $\mathrm{PF}_{6}$ omitted for clarity). Selected bond lengths $(\AA)$ and bond angles $\left({ }^{\circ}\right)$ : Ru-C(1) 2.171(2), Ru-C(2) 2.177(2), Ru-C(3) 2.200(2), Ru-C(4) 2.197(2), Ru-C(5) 2.167(2), Ru-C(6) 2.100(2), Ru-N(3) 2.054(2), Ru-N(4) 2.056(2), C(6)-N(1) 1.357(2), C(6)$\mathrm{N}(2)$ 1.362(2), N(1)-C(7) 1.469(2), N(2)-C(8) 1.475(2), C(7)-C(8) 1.500(2), N(1)-C(9) 1.432(2), N(2)-C(21) 1.436(2); C(6)-Ru-N(3) 83.77(5), C(6)-Ru-N(4) 94.69(5), N(3)-Ru-N(4) 87.44(6), Ru-C(6)-N(1) 126.18(10), Ru-C(6)-N(2) 127.20(10), N(1)-C(6)-N(2) 105.55(12), C(6)-N(1)-C(7) 113.85(12), C(6)-N(2)-C(8) 113.67(12), $\mathrm{N}(1)-\mathrm{C}(7)-\mathrm{C}(8)$ 102.74(13), N(2)-C(8)-C(7) 102.48(12). 
The two compounds $\left[\mathrm{RuCp}\left(\eta^{5}-\mathrm{C}_{5} \mathrm{H}_{4}-\mathrm{CH}_{2}-\mathrm{IPr}\right)\right] \mathrm{PF} \mathrm{F}_{6}$ (3a) and $\left[\mathrm{RuCp}\left(\eta^{5}-\mathrm{C}_{5} \mathrm{H}_{4}-\mathrm{CH}_{2}-\mathrm{SIPr}\right)\right] \mathrm{PF}$ (3b) $(\mathrm{SIPr}=1,3-$ bis(2,6-diisopropylphenyl)-4,5-dihydroimidazol-2-ylidene) form a pair of crystallographically isomorphic and essentially isostructural moieties which differ only in their imidazole rings, for (3a) a imidazole, for (3b) a dihydro-imidazole moiety. Both compounds crystallize triclinic, space group P-1 (no. 2), with similar unit cells and with practically identical asymmetric units, as shown below. Crystallographic and geometric data of both compounds are compared in Table S1. Figures S3 and S4 present views of the Ru-complexes. Further information is provided by the deposited CIF.
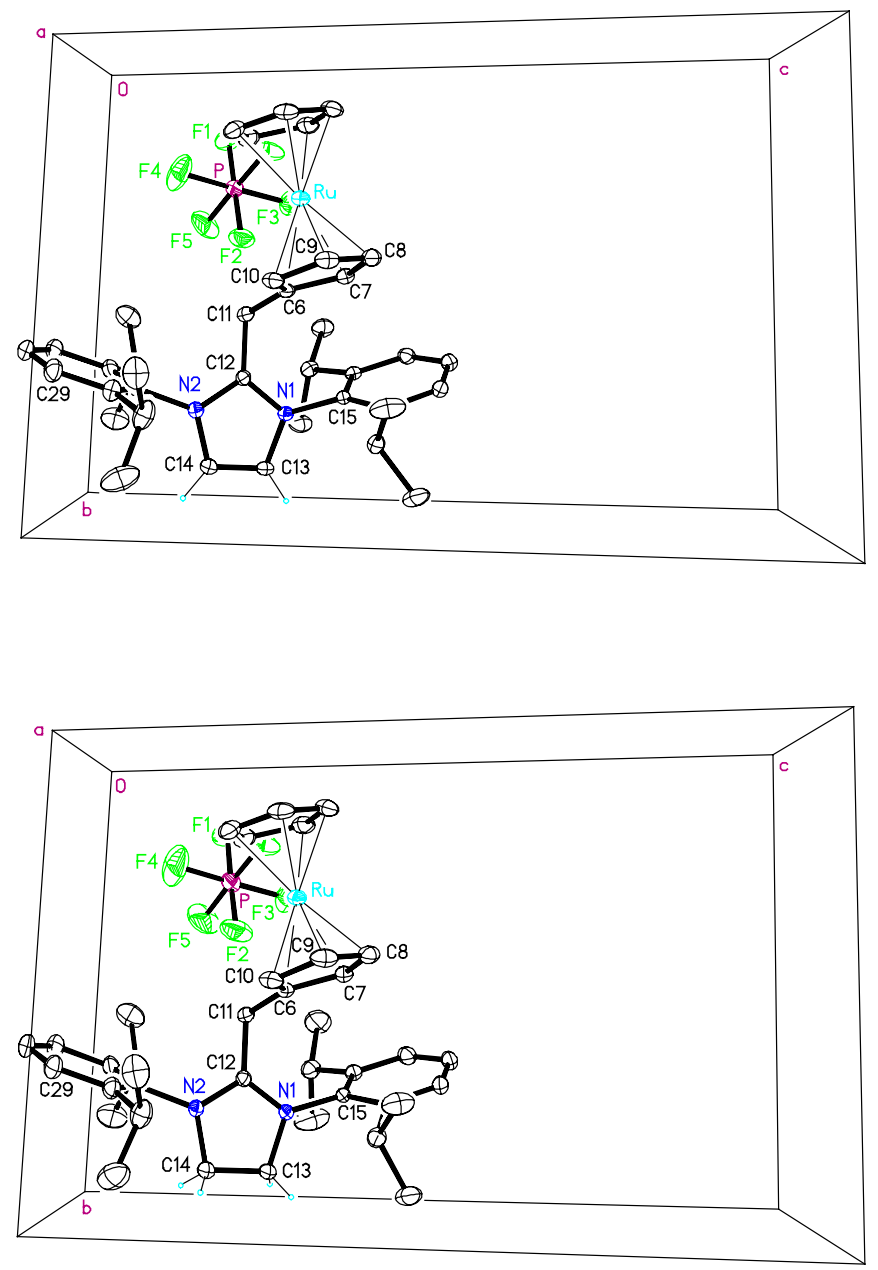

Figure S2. Views of the unit cells and asymmetric units of $\left[\mathrm{RuCp}\left(\eta^{5}-\mathrm{C}_{5} \mathrm{H}_{4}-\mathrm{CH}_{2}-\mathrm{IPr}\right)\right] P F_{6}$ (3a) (top) and $\left[\mathrm{RuCp}\left(\eta^{5}-\mathrm{C}_{5} \mathrm{H}_{4}-\mathrm{CH}_{2}-\mathrm{SIPr}\right)\right] \mathrm{PF}_{6}$ (3b) (bottom). Hydrogen atoms not bonded to the imidazole moieties were omitted for clarity. 
Table S1. Comparison of the two crystallographically isostructural compounds $\left[\mathrm{RuCp}\left(\eta^{5}-\mathrm{C}_{5} \mathrm{H}_{4}-\mathrm{CH}_{2}-\mathrm{IPr}\right)\right] P F_{6}$ (3a) and $\left[\mathrm{RuCp}\left(\eta^{5}-\mathrm{C}_{5} \mathrm{H}_{4}-\mathrm{CH}_{2}-\mathrm{SIPr}\right)\right] \mathrm{PF}_{6}$ (3b).

\begin{tabular}{|c|c|c|}
\hline & $3 a$ & $3 \mathbf{b}$ \\
\hline formula & $\mathrm{C}_{38} \mathrm{H}_{47} \mathrm{~F}_{6} \mathrm{~N}_{2} \mathrm{PRu}$ & $\mathrm{C}_{38} \mathrm{H}_{49} \mathrm{~F}_{6} \mathrm{~N}_{2} \mathrm{PRu}$ \\
\hline fw & 777.82 & 779.83 \\
\hline crystal system & triclinic & triclinic \\
\hline space group & P-1 (no. 2) & P-1 (no. 2) \\
\hline $\bar{a}, \dot{A}$ & $10.3126(9)$ & $10.3582(4)$ \\
\hline $\mathrm{b}, \AA$ & $10.9150(10)$ & $11.0025(5)$ \\
\hline $\mathrm{c}, \AA$ & $16.9277(15)$ & $17.0418(7)$ \\
\hline$\alpha, \operatorname{deg}$ & $87.331(3)$ & $87.587(1)$ \\
\hline$\beta$, deg & $74.758(2)$ & $74.285(1)$ \\
\hline Y, deg & $75.109(2)$ & $75.231(1)$ \\
\hline $\mathrm{V}, \AA^{3}$ & 1776.2(3) & $1807.06(13)$ \\
\hline $\bar{Z}$ & 2 & 2 \\
\hline$\rho_{\text {calc }}, \mathrm{g} \mathrm{cm}^{-3}$ & 1.454 & 1.433 \\
\hline $\mathrm{T}, \mathrm{K}$ & $100(2)$ & $173(2)$ \\
\hline$\mu, \mathrm{mm}^{-1}$ (Mo Ka) & 0.548 & 0.539 \\
\hline$\theta_{\text {max }}$, deg & 30 & 30 \\
\hline no. of rflns measd & 26177 & 26543 \\
\hline no. of unique rflns & 10035 & 10379 \\
\hline no. of rflns $\mid>2 \sigma(I)$ & 9104 & 10177 \\
\hline no. of params / restraints & $433 / 200$ & $433 / 200$ \\
\hline $\mathrm{R}_{1}(\mathrm{I}>2 \sigma(\mathrm{I}))^{[\mathrm{a}]}$ & 0.0316 & 0.0304 \\
\hline $\mathrm{R}_{1}$ (all data) & 0.0363 & 0.0311 \\
\hline $\mathrm{wR}_{2}$ (all data) & 0.0810 & 0.0743 \\
\hline Diff.Four. $\min / \max , \mathrm{e}^{-3}$ & $-0.65 / 0.63$ & $-0.44 / 0.58$ \\
\hline $\mathrm{Ru}-\mathrm{C}(1-5)_{\mathrm{av}} \quad[\AA]$ & $2.183(2)$ & $2.181(2)$ \\
\hline $\mathrm{Ru}-\mathrm{C}(6-10)_{\mathrm{av}}$ & $2.178(2)$ & $2.178(2)$ \\
\hline $\mathrm{C}(6)-\mathrm{C}(11)$ & $1.504(2)$ & $1.505(2)$ \\
\hline$C(11)-C(12)$ & $1.501(2)$ & $1.504(2)$ \\
\hline $\mathrm{C}(12)-\mathrm{N}(1)$ & $1.349(2)$ & $1.326(2)$ \\
\hline $\mathrm{C}(12)-\mathrm{N}(2)$ & $1.349(2)$ & $1.327(2)$ \\
\hline $\mathrm{N}(1)-\mathrm{C}(13)$ & $1.385(2)$ & $1.480(2)$ \\
\hline $\mathrm{N}(2)-\mathrm{C}(14)$ & $1.385(2)$ & $1.481(2)$ \\
\hline$C(13)-C(14)$ & 1.354(2), & $1.517(2)$ \\
\hline $\mathrm{N}(1)-\mathrm{C}(15)$ & 1.452(2), & $1.439(2)$ \\
\hline $\mathrm{N}(2)-\mathrm{C}(27)$ & $1.452(2)$ & $1.444(2)$ \\
\hline $\mathrm{C}(6)-\mathrm{C}(11)-\mathrm{C}(12) \quad[\mathrm{deg}]$ & $114.59(13)$ & 114.31(12) \\
\hline $\mathrm{C}(11)-\mathrm{C}(12)-\mathrm{N}(1)$ & 127.98(13), & $125.50(12)$ \\
\hline $\mathrm{C}(11)-\mathrm{C}(12)-\mathrm{N}(2)$ & 125.19(13), & $123.06(12)$ \\
\hline $\mathrm{N}(1)-\mathrm{C}(12)-\mathrm{N}(2)$ & 106.77(13), & $111.40(12)$ \\
\hline $\mathrm{C}(12)-\mathrm{N}(1)-\mathrm{C}(13)$ & 109.47(12), & $111.02(11)$ \\
\hline $\mathrm{C}(12)-\mathrm{N}(2)-\mathrm{C}(14)$ & 109.69(12), & $111.08(12)$ \\
\hline $\mathrm{N}(1)-\mathrm{C}(13)-\mathrm{C}(14)$ & 107.25(13), & $103.19(12)$ \\
\hline $\mathrm{N}(2)-\mathrm{C}(14)-\mathrm{C}(13)$ & 106.82(13). & $103.00(12)$ \\
\hline
\end{tabular}

\footnotetext{
[a] $\mathrm{R}_{1}=\Sigma|| \mathrm{F}_{\mathrm{o}}|-| \mathrm{F}_{\mathrm{c}}|| \Sigma\left|\mathrm{F}_{\mathrm{o}}\right|, w \mathrm{R}_{2}=\left[\Sigma\left(w\left(\mathrm{~F}_{\mathrm{o}}^{2}-\mathrm{F}_{\mathrm{c}}^{2}\right)^{2}\right) / \Sigma\left(w\left(\mathrm{~F}_{\mathrm{o}}^{2}\right)^{2}\right)\right]^{1 / 2}$
} 


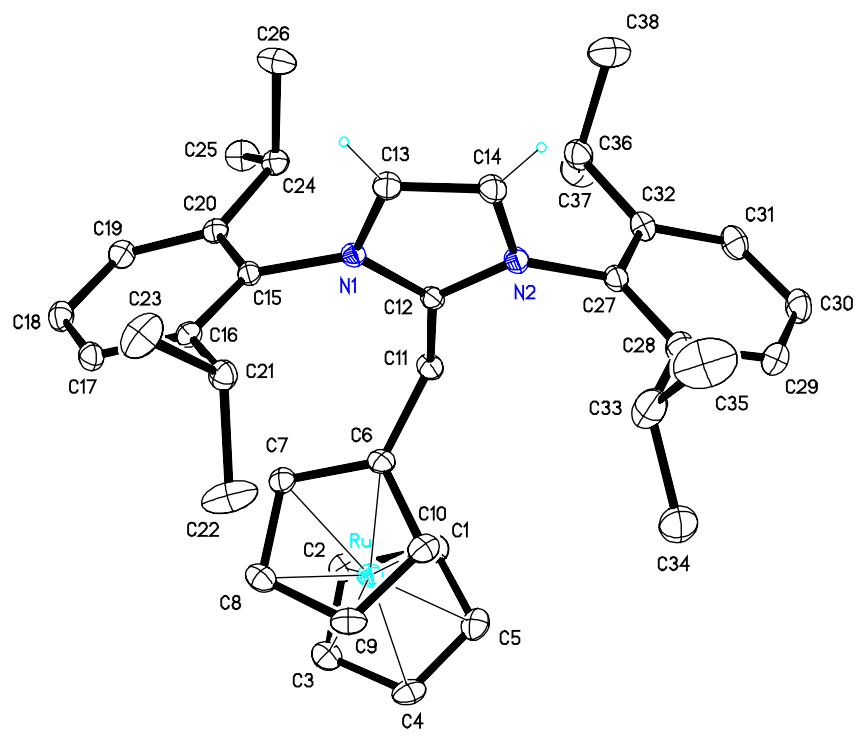

Figure S2. Structural view of $\left[\mathrm{RuCp}\left(\eta^{5}-\mathrm{C}_{5} \mathrm{H}_{4}-\mathrm{CH}_{2}-\mathrm{IPr}\right)\right] \mathrm{PF} \mathrm{F}_{6}$ (3a) showing $40 \%$ thermal ellipsoids. $\mathrm{H}$-atoms shown only for imidazole ring, $\mathrm{PF}_{6}$ omitted for clarity.

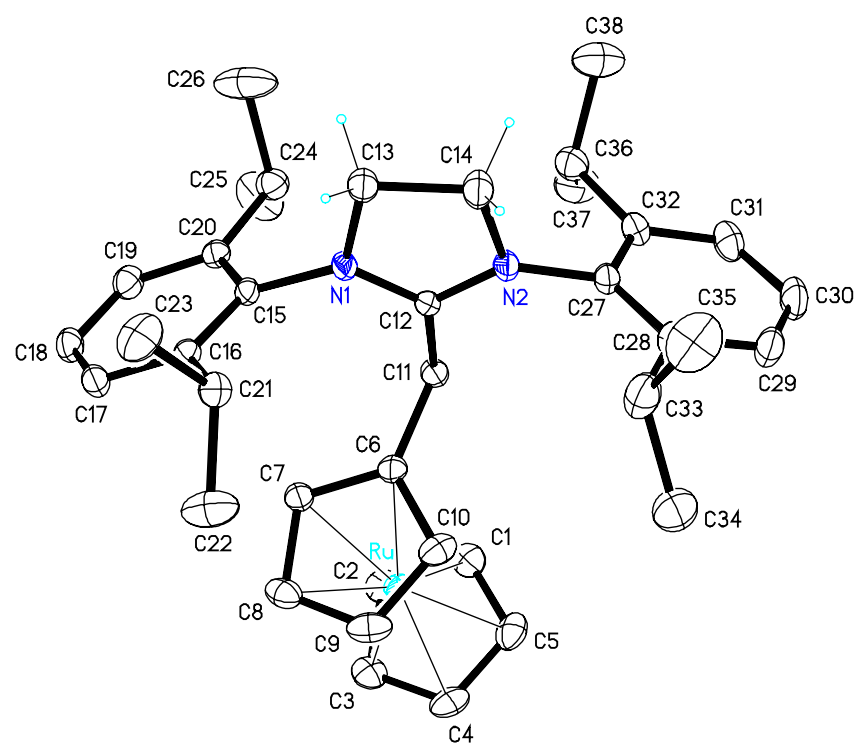

Figure S3. Structural view of $\left[\mathrm{RuCp}\left(\eta^{5}-\mathrm{C}_{5} \mathrm{H}_{4}-\mathrm{CH}_{2}-\mathrm{SIPr}\right)\right] \mathrm{PF}_{6}(3 \mathrm{~b})$ showing $40 \%$ thermal ellipsoids. $\mathrm{H}$-atoms shown only for the dihydroimidazole ring, $\mathrm{PF}_{6}$ omitted for clarity. 